Conference abstract PMS09

\title{
Simulation of Fluid Mixing and Dissolution Processes
}

\author{
T. HöRmANN ${ }^{1}$, M. GsöLL ${ }^{1}$, J. Hofer ${ }^{1}$, D. SUZZI ${ }^{1}$, J. G. KHINAST ${ }^{1,2}$ \\ ${ }^{1}$ Research Center Pharmaceutical Engineering GmbH, Graz, Austria \\ ${ }^{2}$ Institute for Process and Particle Engineering, Graz University of Technology, Graz, Austria \\ E-mail: khinast@tugraz.at (J. G. Khinast)
}

Sci Pharm. 2010; 78: 636

doi:10.3797/scipharm.cespt.8.PMS09

Fluid mixing and dissolution processes are still an area of ongoing research. Numerical simulations have proven to be a valuable tool in understanding and optimizing industrial mixing problems [1]. Computational Fluid Dynamics (CFD) is a well-developed and validated method that is frequently applied in many different fields of applications. Nevertheless, for more complex applications (e. g. industrial mixing) there is still much work left to be done until CFD can be effectively applied as engineering tool providing reliable results in reasonably short calculation times. In this contribution we focus on mixing and dissolution of a bulk powder in a solution inside a stirred tank reactor.

In our work the dissolution kinetics of solids in water were measured in a stirred tank reactor utilising inline Near Infrared (NIR) spectroscopy. The dissolution rate can thus be determined as a function of the powder and process properties such as particle size distribution, solution temperature, impeller speed, and concentrations of the solution.

A dissolution model was derived from the analytical investigations and implemented in the CFD code AVL FIRE. The dissolution test mentioned above was simulated with the CFD method in order to validate the dissolution model.

Due to the fact that real-time CFD simulations of mixing processes are very time-consuming, the dissolution rate of the particles was accelerated in the simulation process and the calculation time was thus reduced. The concentration of a passive scalar is used as a measure for the mass fraction of the dissolving solid particles. This approach allows an analysis of the variation of mixing parameters, and the respective effect on the mixing performance, respectively.

Finally industrial mixing and dissolution processes can be investigated and optimized by applying CFD simulations. In addition, NIR inline measurement techniques enable real-time analysis of the mixing status. Industrial mixing process can thus be controlled by Process Analytical Technology (PAT).

[1] Marshall EM, Bakker A. 2002. Computational Fluid Mixing. Fluent Inc., Lebanon, New Hampshire, USA 